\title{
Falsifying High-Scale Leptogenesis at the LHC
}

\author{
Frank F. Deppisch $*$ and Julia Hard \\ Department of Physics and Astronomy, University College London, \\ London WC1E 6BT, United Kingdom \\ Martin Hirsch \\ AHEP Group, Instituto de Física Corpuscular - C.S.I.C./Universitat de València \\ Edificio de Institutos de Paterna, Apartado 22085, E-46071 València, Spain
}

\begin{abstract}
Measuring a non-zero value for the cross section of any lepton number violating (LNV) process would put a strong lower limit on the washout factor for the effective lepton number density in the early universe at times close to the electroweak phase transition and thus would lead to important constraints on any high-scale model for the generation of the observed baryon asymmetry based on LNV. In particular, for leptogenesis models with masses of the right-handed neutrinos heavier than the mass scale observed at the LHC, the implied large washout factors would lead to a violation of the out-of-equilibrium condition and exponentially suppress the net lepton number produced in such leptogenesis models. We thus demonstrate that the observation of LNV processes at the LHC results in the falsification of high-scale leptogenesis models. However, no conclusions about the viability of leptogenesis models can be drawn from the non-observation of LNV processes.
\end{abstract}

\section{INTRODUCTION}

The observed baryon asymmetry of the universe (BAU), measured in terms of the baryon-to-photon number density ratio [1],

$$
\eta_{B}^{\text {obs }}=(6.20 \pm 0.15) \times 10^{-10},
$$

provides evidence for physics beyond the Standard Model (SM) 2]. A popular scenario for explaining the BAU is through the mechanism of leptogenesis (LG) 3]. In the classic LG scenario, heavy right-handed neutrinos decay out of equilibrium and produce a lepton asymmetry. Necessary ingredients for this process to occur are the presence of $(B-L)$ and $C P$ violation. The produced lepton asymmetry is then rapidly converted into the observed BAU by $(B+L)$-violating sphaleron interactions [4].

Here, we consider lepton number violation (LNV) at the LHC through same sign dilepton signals via resonant processes of the class shown in Fig. 1. The prototype example for this signal is the resonant $W_{R}$ production in left-right symmetric extensions of the SM with heavy Majorana neutrinos [5]. However, generic processes of this kind have been discussed in [6] as tree level high-energy completions of LNV operators that generate neutrinoless double beta $(0 \nu \beta \beta)$ decay 77. For a recent review on $0 \nu \beta \beta$ decay see, for example 8 ]. In Fig. 1, the intermediate particles are different vector or scalar bosons $X$ and $Y^{\left({ }^{\prime}\right)}$ and a fermion $\Psi$. They decay to a final state with four SM fermions composed of two quarks and two same sign charged leptons through unspecified interactions of strengths $g_{i}(i=1,2,3,4)$. In the general case, any combination of quark/anti-quark pairs $u$ and $d$ in the initial state can be realized, and any two of the four final states $f_{i}$ can be leptons. Note that $0 \nu \beta \beta$ constrains only final states with first generation leptons, but at the LHC one could observe LNV in both electrons and muons.
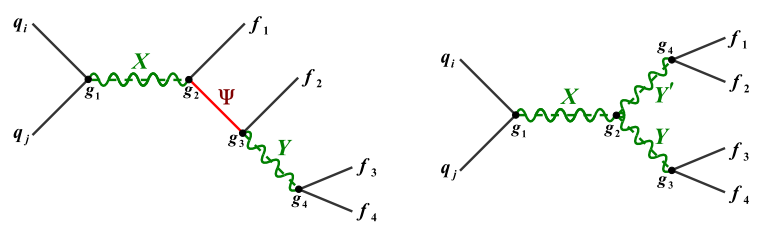

FIG. 1: Possible diagrams contributing to the resonant same sign dilepton signal $p p \rightarrow l^{ \pm} l^{ \pm} q q$ at the LHC. The intermediate particles $X$ and $Y^{(\prime)}$ denote different vector or scalar bosons, $\Psi$ indicates a fermion. In the general case [6, 7], any two of the four fermions $f_{i}$ can be leptons.

In this paper, we explore the consequences of the observation of LNV processes at the LHC on the viability of LG mechanisms. Specifically, we discuss the impact of the observation of LNV at the LHC on the rate of $\Delta L=2$ scattering processes. As we will demonstrate, an observed non-zero cross section can be converted into a lower limit on the washout of the lepton asymmetry in the early universe. If the primordial lepton number asymmetry is originally generated above the LNV scale observed at the LHC, the resulting washout will reduce the asymmetry exponentially, rendering LG ineffective.

We note that the question of falsifying LG at the LHC has been investigated previously in reference [9] within the context of the minimal left-right symmetric model. Our analysis focuses instead on a model-independent approach in which we will derive general limits from the hypothetical observation of the process $p p \rightarrow l^{ \pm} l^{ \pm} q q$.

\section{SAME SIGN DILEPTONS AT THE LHC}

Within the $\Delta L=2$ resonant processes of the form $p p \rightarrow l^{ \pm} l^{ \pm} q q$, we focus on the s-channel diagrams producing a scalar or vector boson $X$ resonantly which then 
cascade decays to the final state $l^{ \pm} l^{ \pm} q q$ through on- or off-shell decays. The parton level cross section can be approximated by a Breit-Wigner resonance

$$
\sigma\left(Q^{2}\right)=\frac{4 \pi}{9}\left(2 J_{X}+1\right) \frac{\Gamma\left(X \rightarrow q_{1} q_{2}\right) \Gamma(X \rightarrow 4 f)}{\left(Q^{2}-M_{X}^{2}\right)^{2}+M_{X}^{2} \Gamma_{X}^{2}},
$$

with $J_{X}$ being the spin of the produced boson and $q_{i}$ indicating the initial partons. The partial decay width $\Gamma(X \rightarrow 4 f)$ describes the complete decay of $X$ as shown in Fig. [1 Integrating over the parton distribution functions (PDFs) in narrow-width approximation of the resonance (2) yields the total LHC cross section [10]

$$
\begin{aligned}
\sigma_{\mathrm{LHC}}= & \frac{4 \pi^{2}}{9 s}\left(2 J_{X}+1\right) \frac{\Gamma_{X}}{M_{X}} f_{q_{1} q_{2}}\left(\frac{M_{X}}{\sqrt{s}}, M_{X}^{2}\right) \\
& \times \operatorname{Br}\left(X \rightarrow q_{1} q_{2}\right) \operatorname{Br}(X \rightarrow 4 f),
\end{aligned}
$$

with the LHC center of mass energy $\sqrt{s}=14 \mathrm{TeV}$ and

$$
\begin{array}{r}
f_{q_{1} q_{2}}\left(r, M^{2}\right)=\int_{r^{2}}^{1} \frac{d x}{x}\left(q_{1}\left(x, M^{2}\right) q_{2}\left(r^{2} / x, M^{2}\right)+\right. \\
\left.q_{2}\left(x, M^{2}\right) q_{1}\left(r^{2} / x, M^{2}\right)\right) .
\end{array}
$$

Here, $q_{i}\left(x, Q^{2}\right)$ is the PDF of parton $q_{i}$ at momentum fraction $x$ and momentum transfer $Q^{2}$. For masses $M \approx$ $1-5 \mathrm{TeV}$, this integral can be well approximated as exponentially decreasing with $M / \sqrt{s}$ [10],

$$
f_{q_{1} q_{2}}\left(\frac{M}{\sqrt{s}}\right) \approx A_{q_{1} q_{2}} \times \exp \left(-C_{q_{1} q_{2}} \frac{M}{\sqrt{s}}\right),
$$

where the coefficients $A_{q q}$ and $C_{q q}$ depend on the combination of the relevant partons $q_{1}, q_{2}$, ranging between $A_{\bar{u} \bar{u}} \approx 200$ to $A_{u u} \approx 4400$ and $C_{u u} \approx 26$ to $C_{\bar{d} \bar{d}} \approx 51$.

\section{LEPTOGENESIS}

The relevant Boltzmann equations for leptogenesis can be generically written in terms of the heavy neutrino and $(B-L)$ number densities per co-moving volume [11] as function of its decay rate $\Gamma_{D}$, the $\mathrm{CP}$ asymmetry $\epsilon$ and the scattering rate $\Gamma_{W}$, which contains inverse $N$ decays as well as any other $\Delta L=1,2$ processes.

The scattering rate $\Gamma_{W}$ induced by the process $q q \leftrightarrow$ $l^{ \pm} l^{ \pm} q q$ is calculated from the reaction density [11]

$$
\gamma\left(q q \leftrightarrow l^{ \pm} l^{ \pm} q q\right)=\frac{T}{32 \pi^{4}} \int_{0}^{\infty} d s s^{3 / 2} \sigma(s) K_{1}\left(\frac{\sqrt{s}}{T}\right),
$$

with the $n$ th-order modified Bessel function $K_{n}(x)$. Here, the process cross section is not averaged over the initial particle quantum numbers. Based on the same underlying process, the washout rate $\Gamma_{W} / H=\left(\gamma / n_{\gamma}\right) / H$ and the LHC cross section $\sigma_{\text {LHC }}$ are directly related. The equilibrium photon density $n_{\gamma} \approx 2 T^{3} / \pi^{2}$ and the Hubble parameter $H \approx 1.66 \sqrt{g_{*}} T^{2} / M_{\mathrm{P}}$ are temperature dependent, with the effective number of relativistic degrees

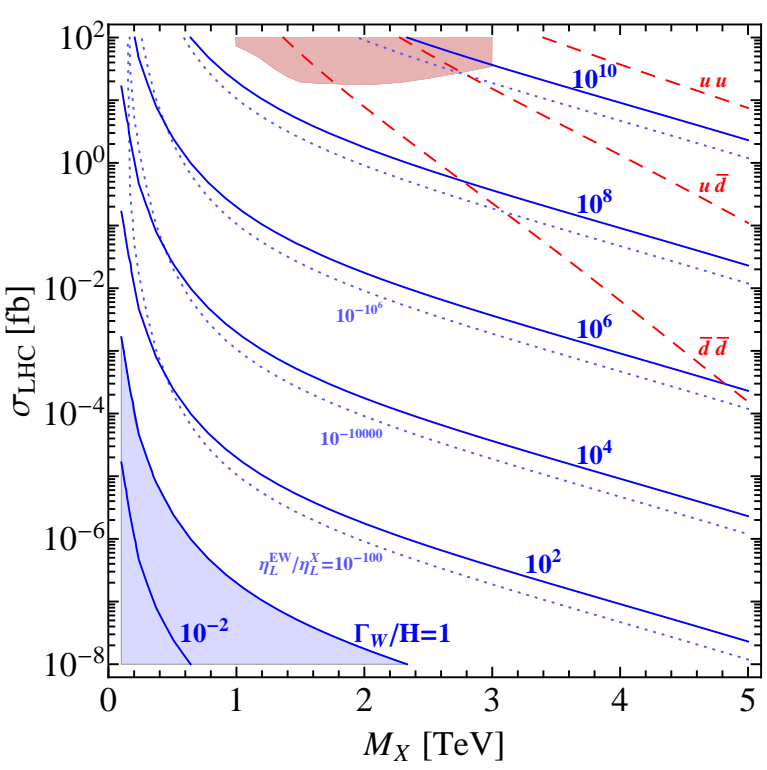

FIG. 2: Washout rate $\Gamma_{W} / H$ at $T=M_{X}$ as a function of $M_{X}$ and $\sigma_{\text {LHC }}$ (solid blue contours). The dotted light blue contours denote the surviving lepton asymmetry at the EW scale relative to its value at $M_{X}, \eta_{L}^{\mathrm{EW}} / \eta_{L}^{X}$. The red dashed curves are typical cross sections of the process $p p \rightarrow l^{ \pm} l^{ \pm} q q$. The red shaded region at the top is excluded due to recent searches for resonant same sign dileptons at the LHC [12].

of freedom $g_{*}(\approx 107$ in the $\mathrm{SM})$ and the Planck mass $M_{\mathrm{P}}=1.2 \times 10^{19} \mathrm{GeV}$. This results in

$$
\frac{\Gamma_{W}}{H}=\frac{0.028}{\sqrt{g_{*}}} \frac{M_{\mathrm{P}} M_{X}^{3}}{T^{4}} \frac{K_{1}\left(M_{X} / T\right)}{f_{q_{1} q_{2}}\left(M_{X} / \sqrt{s}\right)} \times\left(s \sigma_{\mathrm{LHC}}\right),
$$

a relation independent of the branching ratios of the particle $X$ and therefore valid for all coupling strengths $g_{i}$ and also independent of the potential presence of other, lepton number conserving decay modes. Evaluated at $T=M_{X}$, i.e. the approximate onset of the washout process, Eq. (7) yields the order of magnitude estimation

$$
\log _{10} \frac{\Gamma_{W}}{H} \gtrsim 6.9+0.6\left(\frac{M_{X}}{\mathrm{TeV}}-1\right)+\log _{10} \frac{\sigma_{\mathrm{LHC}}}{\mathrm{fb}},
$$

using the conservative values $A_{q q}=5000$ and $C_{q q}=26$ for Eq. (5). From this approximation alone it is clear that the observation of the resonant process $p p \rightarrow l^{ \pm} l^{ \pm} q q$ at the LHC corresponds to a very strong washout of the lepton asymmetry in the early universe. For example, the observation of a resonance at $M_{X} \approx 2 \mathrm{TeV}$ with a cross section $\sigma_{\mathrm{LHC}} \approx 1 \mathrm{fb}$ corresponds to $\Gamma_{W} / H \approx$ $3 \times 10^{7}$. The exact relation (7) is shown in Fig. 2, based on the smallest washout among all parton combinations. For any realistic cross section observable at the LHC with $\sigma_{\mathrm{LHC}} \gtrsim 10^{-2} \mathrm{fb}$, the resulting lepton number washout in the early universe is always highly effective $\left(\Gamma_{W} / H \gg 1\right)$. The dashed curves, for example, show 
typical cross sections for different parton combinations in the case of a particle $X$ with gauge-strength total width, $\Gamma_{X} / M_{X}=g^{2} /(32 \pi)$, with $g=0.5$ and branching ratios $\operatorname{Br}\left(X \rightarrow q_{1} q_{2}\right)=\operatorname{Br}(X \rightarrow 4 f)=0.5$. Without a source regenerating the lepton asymmetry below $M_{X}$, the washout leads to an exponential suppression of the surviving asymmetry at the EW scale compared to the value present at $M_{X}, \eta_{L}^{\mathrm{EW}} / \eta_{L}^{\mathrm{X}} \approx \exp \left(-\Gamma_{W} / H\right)$. This suppression is also shown in Fig. 2 highlighting that the observation of LNV processes at the level $\sigma_{\mathrm{LHC}} \gtrsim 10^{-2} \mathrm{fb}$ would necessarily result in an enormous washout of any pre-existing lepton asymmetry.

It should be stressed again that the above analysis is highly model independent and purely based on the observables $M_{X}$ and $\sigma_{\mathrm{LHC}}$ of the process. The approximations used in our calculation, such as the narrow-width resonance assumption, are not expected to change this conclusion in any way. In fact, the direct relation between the LHC cross section and the washout rate is expected to hold for any LNV process, with a proportionality only affected by the kinematics of the process.

In order to further assess the impact of the resulting washout, we calculate the baryon asymmetry in the standard LG scenario with one heavy neutrino $N$, neglecting all other washout reactions. The Boltzmann equations in this case are most compactly expressed in terms of the out-of-equilibrium heavy Majorana neutrino density deviation $\delta \eta_{N}=\eta_{N} / \eta_{N}^{\mathrm{eq}}-1$ and the lepton density $\eta_{L}=n_{L} / n_{\gamma}$ normalized to the photon density $n_{\gamma}$ [13],

$$
\begin{aligned}
\frac{d \delta \eta_{N}}{d z} & =\frac{K_{1}\left(r_{N} z\right)}{K_{2}\left(r_{N} z\right)}\left[r_{N}+\left(1-r_{N}^{2} K_{D} z\right) \delta \eta_{N}\right], \\
\frac{d \eta_{L}}{d z} & =\epsilon K_{D} r_{N}^{4} z^{3} K_{1}\left(r_{N} z\right) \delta \eta_{N}-K_{W} z^{3} K_{1}(z) \eta_{L},
\end{aligned}
$$

with the decay factor $K_{D}=\Gamma_{D} / H$ at $T=M_{N}$ and the washout factor $K_{W}=\Gamma_{W} / H$ at $T=M_{X}$. We define the evolution parameter $z=M_{X} / T$. The above Boltzmann equations explicitly contain the full temperature $(z)$ dependence with the hierarchy between the masses of $N$ and $X$ given by $r_{N}=M_{N} / M_{X}$. Note that Eqs. (9) and (10) implicitly assume that the $C P$ asymmetry is generated from the decay of the heavy neutrino. Other mechanisms will yield similar results.

Fig. 3] shows a typical solution to the Boltzmann equations (91) and (10) with $M_{N}=1.5 \mathrm{TeV}, M_{X}=2 \mathrm{TeV}$, $C P$ asymmetry $\epsilon=10^{-2}, K_{D}=10^{8}$, and $K_{W} \approx 6 \times 10^{6}$ (smallest washout corresponding to $\sigma_{\mathrm{LHC}}=0.1 \mathrm{fb}$ ). For sufficiently strong rates $K_{D}$ and $K_{W}$, the linear dropoff behaviour depicted can be approximated as $\delta \eta_{N}=$ $1 /\left(r_{N} K_{D} z\right)$ and $\eta_{L}=r_{N}^{2} \epsilon /\left(K_{W} z\right)$, respectively, in which case $\eta_{L}$ is generally independent of $K_{D}$ [13]. For $M_{N}=$ $M_{X}, \eta_{L}$ will eventually freeze-out to a constant value, but for a different hierarchy, the contributions to $\eta_{L}$ depend differently on the temperature. In the case $r_{N}<1$, shown in the figure, heavy neutrino decays can not be compensated by the washout for $z>1$, whereas for $r_{N}>$

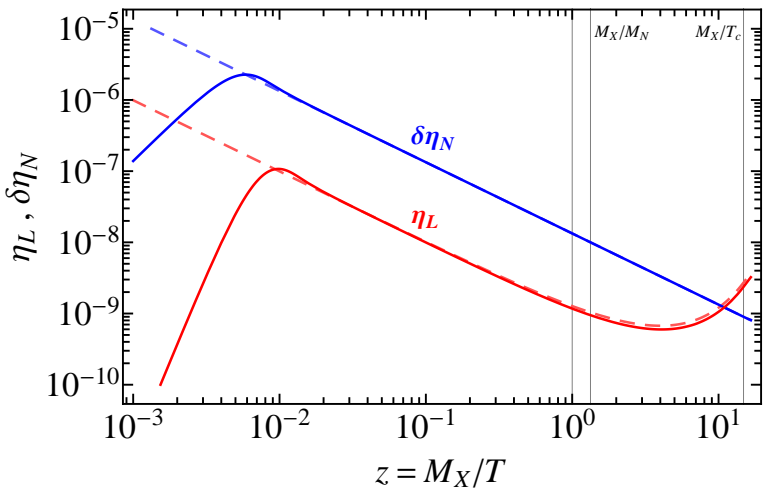

FIG. 3: Solution to the Boltzmann equations for $M_{N}=$ $1.5 \mathrm{TeV}, M_{X}=2 \mathrm{TeV}, C P$ asymmetry $\epsilon=10^{-2}, K_{D}=10^{8}$ and $K_{W} \approx 6 \times 10^{6}$ (smallest washout corresponding to $\sigma_{\text {LHC }}=0.1 \mathrm{fb}$ ). The dashed lines denote approximated solutions discussed in the text.

1 the washout is always effective for $z>1$ leading to an over-proportional drop-off. The general behaviour can be well approximated by $\eta_{L} \approx r_{N}^{2} \epsilon /\left(K_{W} z\right) \exp \left(\left(1-r_{N}\right) z\right)$. In Fig. 3, this approximation is shown as a red dashed curve. Such a behaviour is not realistic in a given model where other processes are expected to contribute, but allows to draw model-independent conclusions. Most importantly we neglect washout from processes mediated by the heavy neutrino driven by the same interaction(s) that generate the lepton asymmetry. Taking into account all washout processes in a consistent fashion will guarantee a well-behaved freeze-out behaviour for $\eta_{L}$. In this spirit, our solution for $\eta_{L}$ can be considered as a conservative, model-independent but also possibly weak upper limit on the generated lepton asymmetry.

The conversion of the lepton number to the final baryon asymmetry can be calculated by $\eta_{B}=$ $-d_{\mathrm{rec}} r_{B / L} \eta_{L}\left(T_{c}\right)$ with $r_{B / L}=\left(8 N_{g}+4 N_{H}\right) /\left(14 N_{g}+\right.$ $\left.9 N_{H}\right) \approx 1 / 2$ in a general theory with $N_{g}$ fermion generations and $N_{H}$ Higgs doublets 14]. The critical temperature of the electroweak phase transition is denoted by $T_{c} \approx 135 \mathrm{GeV}, d_{\mathrm{rec}} \approx 1 / 27$ (in the $\mathrm{SM}$ ) describes the increase of the photon density during the recombination epoch, and $\eta_{L}\left(T_{c}\right)$ is the lepton asymmetry at the sphaleron decoupling temperature. For details see [15].

We arrive at an upper limit for the baryon asymmetry,

$$
\left|\eta_{B}\right| \lesssim \frac{M_{N}^{2}}{M_{X}^{2}} \frac{T_{c}}{M_{X}} \frac{r_{B / L} d_{\mathrm{rec}}|\epsilon|}{K_{W}\left(M_{X}, \sigma_{\mathrm{LHC}}\right)} e^{\left(M_{X}-M_{N}\right) / T_{c}}
$$

that can be compared to the observed value (11). By using the derived approximation (8), we can set an upper limit on the baryon asymmetry as a function of the LG parameters $m_{N}$ and $\epsilon$, and the observables $M_{X}$ and $\sigma_{\mathrm{LHC}}$ 


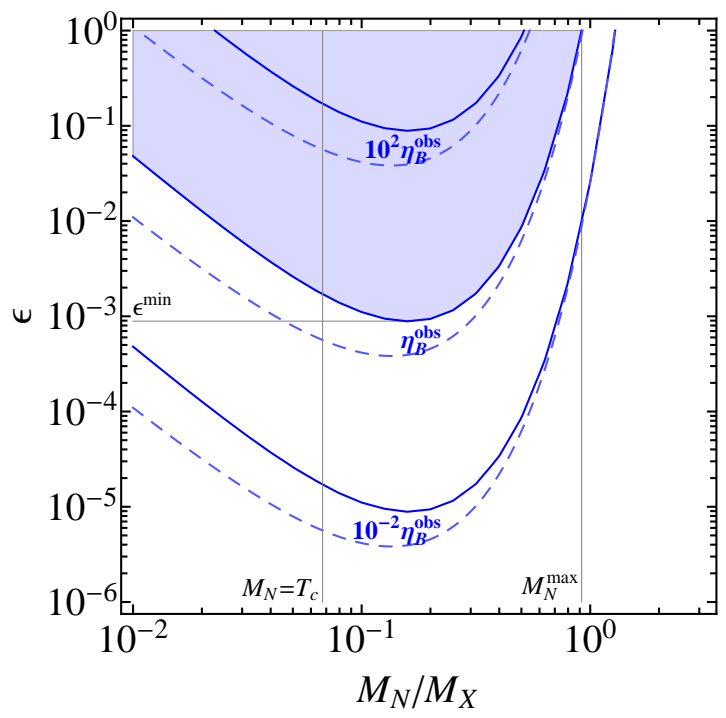

FIG. 4: Baryon asymmetry $\eta_{B}$ as a function of $M_{N} / M_{X}$ and $\epsilon$ for $M_{X}=2 \mathrm{TeV}$ and $\sigma_{\mathrm{LHC}}=0.1 \mathrm{fb}$ (solid contours). The intermediate contour corresponds to the observed value $\eta_{B}^{\text {obs }}$, the other two contours give 100 times higher and lower values, respectively. Correspondingly, the dashed contours are determined using the approximation Eq. (11).

of the LHC process,

$$
\begin{aligned}
\log _{10}\left|\frac{\eta_{B}}{\eta_{B}^{\mathrm{obs}}}\right| & \lesssim 2.4 \frac{M_{X}}{\mathrm{TeV}}\left(1-\frac{4}{3} \frac{M_{N}}{M_{X}}\right) \\
& +\log _{10}\left[|\epsilon|\left(\frac{\sigma_{\mathrm{LHC}}}{\mathrm{fb}}\right)^{-1}\left(\frac{4}{3} \frac{M_{N}}{M_{X}}\right)^{2}\right] .
\end{aligned}
$$

In Fig. 4 the resulting baryon asymmetry for both the exact solution of the Boltzmann equations (solid line) as well as the approximation (11) (dashed line) is shown as a function of $r_{N}=M_{N} / M_{X}$ and $\epsilon$ for $M_{X}=2 \mathrm{TeV}$ and $\sigma_{\mathrm{LHC}}=0.1 \mathrm{fb}$. Two important conclusions can be drawn: (i) For $M_{N}>M_{N}^{\max } \approx M_{X}$ it is not possible to generate a large enough baryon asymmetry. As our calculation gives a conservative upper limit for $\eta_{B}$, this means that the observation of the LNV process at the LHC excludes high energy LG models. (ii) For $M_{N}<M_{X}$ there exists a lower limit on the $C P$ asymmetry $\epsilon>\epsilon^{\text {min }} \approx 10^{-3}$, which strongly constrains resonant LG models.

\section{DISCUSSION AND CONCLUSIONS}

We have discussed the impact of a possible observation of lepton number violation at the LHC on leptogenesis. We have shown that for right-handed neutrinos heavier than the mass scale at which LNV is observed at the LHC, the resulting washout factor will reduce any preexisting lepton asymmetry $L$ exponentially, rendering LG ineffective. Our arguments should be generally valid and
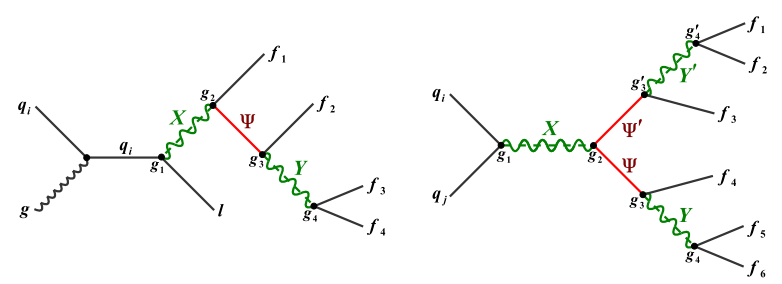

FIG. 5: Further example diagrams for different LNV process classes at the LHC with 5 (left) and 6 (right) final state particles. Note that in the latter case, in addition to the $q q$ initial state shown, also gluon-gluon initial state diagrams can exist.

not depend on the particular realization of LG, although we have concentrated on the "standard" scenario with right-handed neutrinos. Thus, high-scale thermal leptogenesis models can be falsified in case of the observation of LNV processes at the LHC. However, it should be stressed that no conclusions about the viability of LG can be drawn from the non-observation of these processes.

There are a few possible caveats to consider: (i) One could imagine a situation where LNV is generated in the early universe in the third family only. As there is no experimental proof so far that $e^{ \pm} e^{ \pm} \leftrightarrow \tau^{ \pm} \tau^{ \pm}$was in equilibrium in the early universe, the observation of LNV at the LHC for only $e$ and/or $\mu$ is not sufficient in this case. A non-zero observation of $p p \rightarrow l^{ \pm} l^{ \pm} q q$ for either $l l=e e, \mu \mu$ and $\tau \tau$, or for $e \mu$ and $e(\mu) \tau$ is necessary to unambiguously falsify LG models in the way presented. (ii) Our discussion leaves open the possibility of LG with $M_{N}<M_{X}$. Resonant sub-TeV scale LG with large $C P$ asymmetry $\epsilon$, for example, would still be possible. This loophole is not limited to the classical LG with righthanded neutrinos. In general, any mechanism producing a "hidden" lepton number which is converted to $B$ below $M_{X}$, would not be ruled out. Nevertheless, it is still possible to derive a large model-independent lower limit on the required $C P$ asymmetry. (iii) $\mathrm{SM}$ sphaleron processes only affect electroweak fermion doublets, but left- and right-handed fermions are in thermal equilibrium around the electroweak scale for Yukawa couplings larger than $\approx 10^{-8}$. This is the case for all charged leptons. Our conclusions therefore also apply if the LNV process at the LHC involves right-handed leptons.

Finally, although we concentrated on the resonant process of Fig. 1. we believe our argumentation can be easily adapted to other cases. For example, leptoquark production can occur as in Fig. 5 (left) and pair production of heavy states as in Fig. [5 (right). We conjecture that our relation between the observation of the LNV at LHC and the lower limit on the washout factor is valid for these cases too, most likely even with larger numerical factors. The discovery of LNV at the LHC could then have profound implications, especially if combined with the observation of $0 \nu \beta \beta$ decay at a rate expected from a short range operator induced by a process as in Fig. 1. Such 
an experimental scenario would dis-favour the standard high scale seesaw mechanism as both leptogenesis and the dominance of light Majorana neutrinos mediating $0 \nu \beta \beta$ decay are rendered ineffective.

\section{ACKNOWLEDGMENTS}

The work of FFD and JH was supported partly by the London Centre for Terauniverse Studies (LCTS), using funding from the European Research Council via the Advanced Investigator Grant 267352. The work of MH was supported by the Spanish MINECO under grants FPA2011-22975 and MULTIDARK CSD200900064 (Consolider-Ingenio 2010 Programme), by Prometeo/2009/091 (Generalitat Valenciana), and by the EU ITN UNILHC PITN-GA-2009-237920. FFD and JH would like to thank Robert Thorne for useful discussions.

* Electronic address: f.deppisch@ucl.ac.uk

$\dagger$ Electronic address: j.harz@ucl.ac.uk

¥ Electronic address: mahirsch@ific.uv.es

[1] P. A. R. Ade et al. [Planck Collaboration], arXiv:1303.5076 [astro-ph.CO].
[2] W. Buchmuller, R. D. Peccei, T. Yanagida, Ann. Rev. Nucl. Part. Sci. 55 (2005) 311.

[3] M. Fukugita, T. Yanagida, Phys. Lett. B 174 (1986) 45.

[4] V. A. Kuzmin, V. A. Rubakov, M. E. Shaposhnikov, Phys. Lett. B 155 (1985) 36.

[5] W.-Y. Keung, G. Senjanovic, Phys. Rev. Lett. 50, 1427 (1983); S. P. Das, F. F. Deppisch, O. Kittel, J. W. F. Valle, Phys. Rev. D 86 (2012) 055006; T. Han, I. Lewis, R. Ruiz and Z.-g. Si, Phys. Rev. D 87, 035011 (2013) [Erratum-ibid. D 87, no. 3, 039906 (2013)].

[6] J. C. Helo, M. Hirsch, H. Päs, S. G. Kovalenko, Phys. Rev. D 88 (2013) 073011.

[7] F. Bonnet, M. Hirsch, T. Ota, W. Winter, JHEP 1303, 055 (2013).

[8] F. F. Deppisch, M. Hirsch, H. Päs, J. Phys. G 39, 124007 (2012).

[9] J.-M. Frere, T. Hambye, G. Vertongen, JHEP 0901 (2009) 051.

[10] A. Leike, Phys. Rept. 317 (1999) 143.

[11] G. F. Giudice, A. Notari, M. Raidal, A. Riotto, A. Strumia, Nucl. Phys. B 685 (2004) 89.

[12] CMS Collaboration [CMS Collaboration], CMS-PASEXO-12-017.

[13] F. F. Deppisch, A. Pilaftsis, Phys. Rev. D 83 (2011) 076007.

[14] S. Y. Khlebnikov, M. E. Shaposhnikov, Nucl. Phys. B 308 (1988) 885.

[15] A. Pilaftsis, T. E. J. Underwood, Phys. Rev. D 72 (2005) 113001. 